\title{
Prosthetic Stage Of Anterior Maxillary Implant- A Case Study
}

\author{
Durafshan Rehman, Saqib Rashid
}

\begin{abstract}
:
One of the greatest challenges for implant placement in the maxillary anterior zone is not only the restoration of function but also the esthetics. The reestablishment of a normal alveolar contour after implant placement is a critical step in esthetic success. This important aspect consists of replacing the lost portion of the alveolar process and associated soft tissue. It can be onerous to establish a balance between the tooth and the surrounding apparatus at prosthesis - soft tissue interface. This case presents the implant placement aspect with an in-depth consideration of positional parameters and post implant placement with consideration of soft tissue management and manipulation. Thus, the final restoration fitted precisely and harmoniously with the natural and esthetic contours of the gingiva and provided the esthetic results which both the practitioner and the patient were aiming to achieve. Hence, validating use of this technique for suitable patients.
\end{abstract}

Keywords: Anterior implant, Esthetic zone, Prosthetic stage, Immediate loading, Restoration guided.

\section{INTRODUCTION:}

Determining the correct position of implant to provide the patient with a restoration that creates the illusion of a natural tooth comes with numerous challenges including the position of gingival papillae, gingival symmetry and emergence profile of the restoration that replicates the natural tooth.

To provide the patient with an esthetic restoration the most important part is a detailed workup including history and examination. The data collection must focus on patient's medical and dental history, extra and intra oral examinations, radiographic analysis, patient's expectations and implant failure risks (esthetic or functional). For a functional and esthetic implant, a facial, dental and periodontal examination should be conducted including periodontal charting and radiographic analysis. The gingival biotype should be assessed by classifying it into thick or thin accordingly as this will help assessment of postsurgical recession. Also determine the vertical height of the interproximal bone and the edentulous site as the presence of bone will influence establishment of overlying soft tissue.

The two major aspects of this case are immediate loading implant and the tissue manipulation after implant placement. According to Wohrle's data, if an immediate implant was placed the soft tissue loss never exceeded $1.5 \mathrm{~mm}$ for any restoration and Wohrle considered that the harmony and continuity of the hard and soft tissues were predictably achieved in all cases. Wohrle reported

I Durafshan Rehman
I Registrar, Department of Prosthodontics,
I Bahria University Medical And Dental College Karachi.
I Saqib Rashid
I Fead of Department Operative Dentistry
I Iatima Jinnah Dental College Karachi.
I Received: 25-10-17
I Accepted: 22-01-18
|

the success of 14 consecutive cases in which he extracted non restorable teeth, placed tapered implants with various surfaces and restored them with provisional restorations on the same day. It should be noted that none of the restorations had any occlusal contacts and patients were instructed to avoid using the implants and restorations for periods up to six months postoperatively. The second most important aspect of this case is to provide with a provisional restoration so that the tissue is not left unsupported. With the help of a provisional restoration we can manage and manipulate the healing soft tissue collar around the implant. A provisional restoration helps to develop a gingival tissue form of a tooth, as the implant restoration emerges from the sulcus.

This article presents a case of immediate dental implant placement into fresh extraction socket in the esthetic zone followed by the prosthetic management and final restoration. The case selection, treatment planning, surgical protocol and restorative outcomes are presented and discussed.

\section{CASE REPORT:}

A thirty-eight year old male patient was referred to our clinic to replace mobile maxillary left central incisor. Patient's chief complaint was his compromised esthetics. He had no systemic medical problems and no parafunctional habits. Oral hygiene was maintained. He was previously under treatment of another dentist where he reported with complaint of mobility in upper anterior teeth and halitosis. He was diagnosed with generalized periodontitis and the treatment was started immediately. His teeth were stabilized by splinting the grade II mobile maxillary left central incisor with the right maxillary central incisor. Regular visits of periodontal therapy were done for three months. When there were no signs of active progression of periodontitis, patient was referred to our clinic for replacement of mobile maxillary left central incisor. To make sure that the patient was a candidate for implant, a detailed workup was done which 
included the extraoral and intraoral examination. Patient had an adequate lip support, low smile line but a thin gingival biotype which is a challenge to restore as it is less resistant to trauma and more prone to recession.

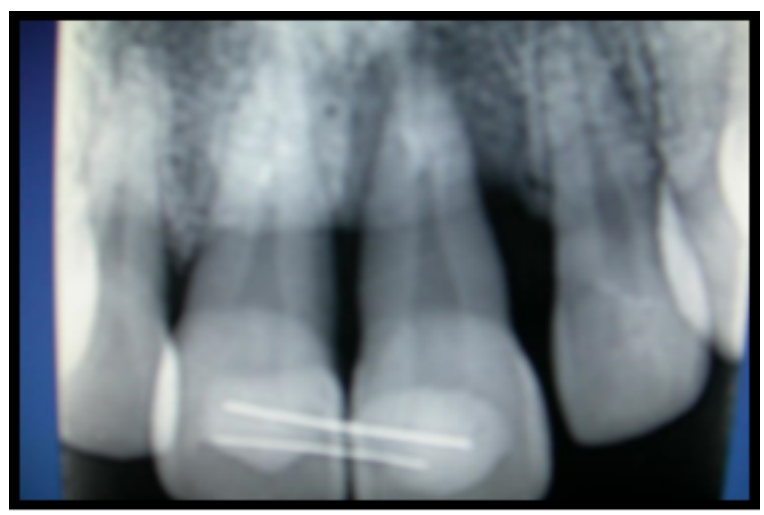

Fig 1 Pre-operative radiograph

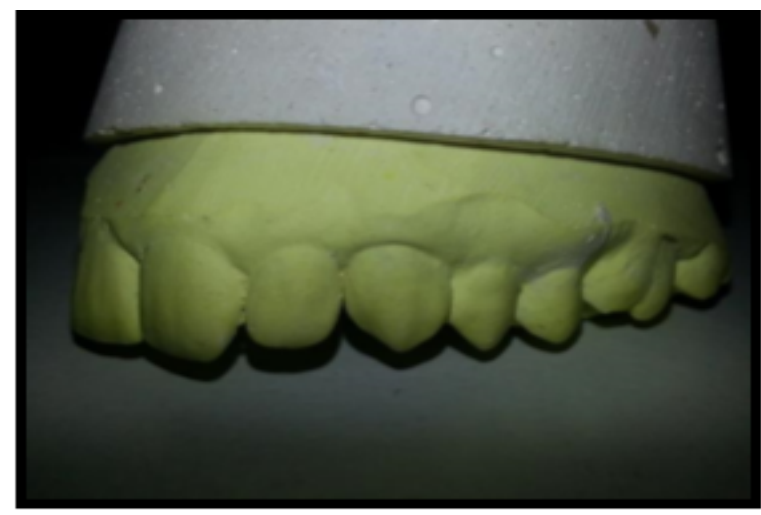

Fig 2(A) Pre-operative model lateral view

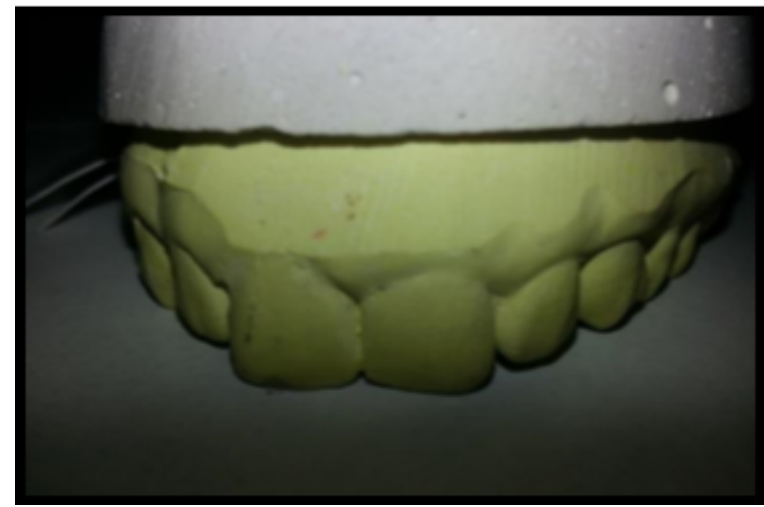

Fig 2(B) Pre-operative model frontal view

Fig 1 shows the diagnostic radiograph with a generalized bone loss due to a previous history of periodontitis. Splinting of both central incisors can also be seen. Patient was advised removal of maxillary left central incisor followed by immediate implant placement. Impressions were taken, models were duplicated Fig 2(A \& B) and mock-up was done for the tooth to be replaced by implant with an acrylic tooth. Keeping in mind the vertical and horizontal parameters of implant placement initial drill was used and surgical template was fabricated. An atraumatic extraction was performed. Due to resorbed buccal cortical plate, the surgical site needed bone as well as soft tissue augmentation due to the presence of thin

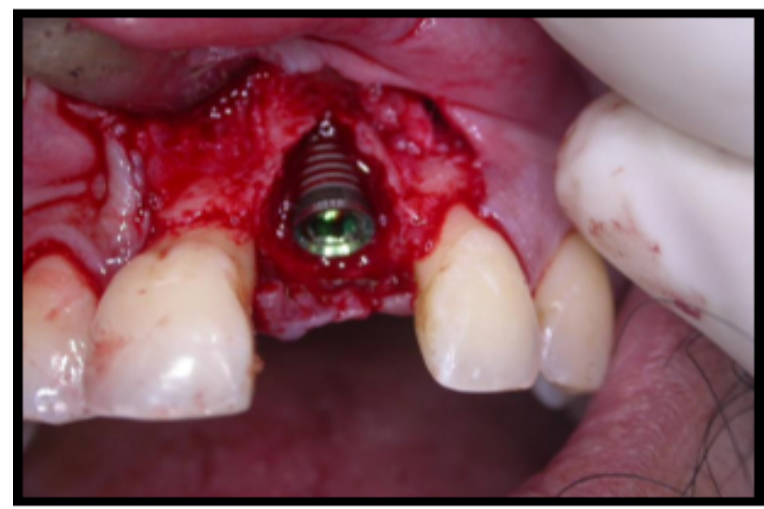

Fig 3 Implant placed (size 4.0x12mm)

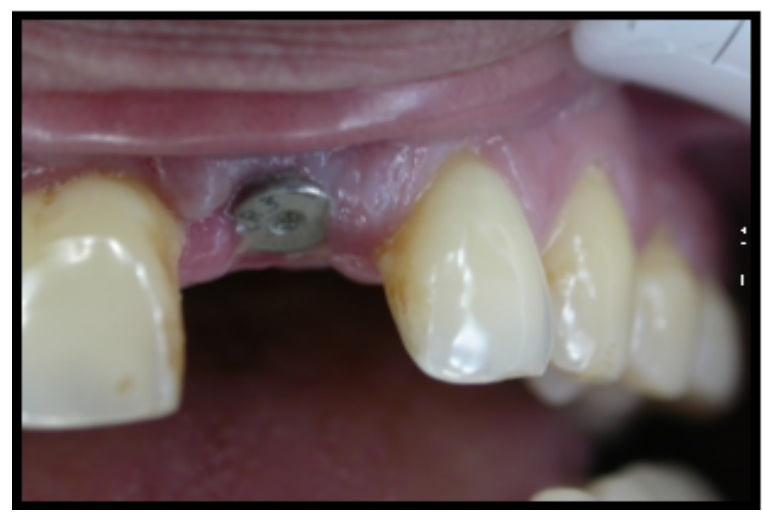

Fig 4 Clinical view after 12 weeks

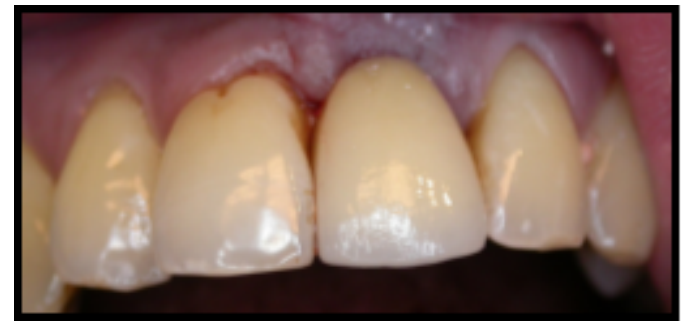

Figure-: 5 Final Crown Cemented

gingival biotype as mentioned earlier.

The vertical aspect (depth contour and implant angulation) and horizontal aspect (amount of bone available bucco lingually and mesio distally) are significant parameters which should be considered for implant placement, so that a sufficient amount of bone on all sides of the implant is available.

After implant placement 'freeze dried cortical bone allograft' FDBA (SureOss) was placed. It promotes bones regeneration through osteoconductive and osteoinductive actions and retains the growth factors, proteins and minerals. Site was then covered by skin graft (SureDerm). 
It is natural human skin tissue. It is a dermal allograft that has been freeze-dried, after the cells that cause immune response have been removed from the epidermis and the dermis.

The patient was recalled at regular intervals to observe the healing process. Fig 4 shows the clinical picture of the site after 12 weeks, depicting nicely healed implant collar. Percussion test was also performed before proceeding to the prosthetic stage.

As the implant healed and the surrounding tissue was also healed, we removed the healing screw. The abutment was placed and necessary crown preparation was done. Then by placement of screw and transfer coping all the hard and soft tissues were recorded with the impression material (IMPREGUM) without any discrepancies.

During the laboratory stages of fabrication, the crown margins were adapted to the cast so that the crown fitted the gingival tissue perfectly once placed inside the patient's oral cavity, leaving no open margins and matching the emergence profile of the patient's adjacent teeth. Fig 5 shows final restoration in place, with no black triangles and the gingival contours matching that of the adjacent teeth.

\section{DISCUSSION:}

Considering the restoration as the coronal extension of implant, this case is handled on the principal of restoration guided implant placement rather than conventional bone guided implant placement. This procedure mandates that the implant is placed where it can be properly restored. If the site is lacking the bone or soft tissues, then, augmentation procedures must be employed to create an acceptable site. Prosthesis designing is also a major step in implant placement as the esthetic outcome is dependent on it. Systematic reviews of the survival and complications of implants and associated prostheses identified 6 categories of complications: surgical complications, implant loss, bone loss, peri-implant softtissue complications, mechanical complications and esthetic/phonetic complications. Even if implant is properly osteointegrated, failure of managing soft tissues properly can lead to esthetic failure of implant.

Following the implant placement, if recession of the soft tissue margin is observed and attributed to bone and soft tissue remodeling, the connective tissue and the junctional epithelium will migrate apically beyond the implantabutment interface. In a 5-year multicenter study, Henry and colleagues reported an implant success rate of about $96 \%$ for single-tooth replacements in the anterior maxilla. However, they also reported an esthetic failure rate of about 9\% for implant placement in this area. Vertical (apicocoronal) bone loss is usually the result of periodontal disease and represents a particularly difficult challenge for implant placement. No surgical approach is available to predictably augment the ridge height. Some case reports suggest a surgical approach using nonresorbable membrane. When a horizontal defect in the socket buccal wall is present, the size should be determined. If this defect is less than $5 \mathrm{~mm}$ in the apicocoronal direction or less than one third of the mesiodistal dimension between the adjacent teeth, immediate implant placement at the time of extraction can be accomplished. Depending on the size of the dehiscence, lateral bone augmentation or guided bone regeneration maybe performed as needed.

\section{CONCLUSION:}

This case report presents implant placement protocols and parameters to improve esthetic outcomes of implants in anterior maxillary region. It further emphasizes on soft tissue management after the implant placement to prevent gingival recession leading to black triangles and esthetic failures. The procedure mentioned here for prosthetic stage management of dental implant in anterior maxilla can reduce the percentage of aesthetic failures in implant candidates and provide them with best possible outcome.

\section{REFERENCES:}

1. F. B. Patient selection for dental implants. Part 1: data gathering and diagnosis. J Indiana Dent Assoc. 2000; 79(1):8-11.

2. MG SALG Periodontal implications in implant treatment planning for aesthetic results.. Pract Periodontics Aesthet Dent. 1998; 10(5):655-64 [quiz: 666].

3. Jansen CE WA. Presurgical treatment planning for the anterior single-tooth implant restoration.. Compend Contin Educ Dent. 1995; 16(8):746, 748-52, 754 passim [quiz: 764].

4. JC. K. Predictable single tooth peri-implant esthetics: five diagnostic keys. Compend Contin Educ Dent. 2001; 22(3):199-206 [quiz: 208].

5. Belser U BDHF. Consensus statements and recommended clinical procedures regarding esthetics in implant dentistry. Int J Oral Maxillofac Implants. 2004; 19(Suppl):73-4..

6. Kan JY RKUKea. Dimensions of peri-implant mucosa: an evaluation of maxillary anterior single implants in humans. J Periodontol. 2003; 74(4):557-62.

7. PS. W. Single-tooth replacement in the aesthetic zone with immediate provisionalization: Fourteen consecutive case reports. Pract Periodont Aesth Dent. 1998; 10 (9):11071114.

8. Cobb et al.. Macintosh and Sutherland, 2004. 1998.

9. Goodacre CJ BGRKea. Clinical complications with implants and implant prostheses. J Prosthet Dent. 2003; 90(2):121-32.

10. Abdulhadi Warreth HF, R M, D M. An Introduction to Single Implant Abutments Dent Update. 2013; 40: 1-7. https:/ / doi.org/ 10.12968/ denu.2013.40.1.7

11. Henry PJ, L, W, J Tea. Osseointegrated implants for singletooth replacement: a prospective 5-year multicenter study. 
Int J Oral Maxillofac Implants. 1996; 11(4):450-5.

12. Jovanovic SA AR. Reconstruction of the posterior maxilla following total loss of crestal bone support. Pract Periodontics Aesthet Dent. 1998; 10(2):241-4.

13. Chiapasco MASREea. Clinical outcome of autogenous bone blocks or guided bone regeneration with e-PTFE membranes for the reconstruction of narrow edentulous ridges. Clin Oral Implants Res. 1999; 10(4):278-88.

14. Salama H SM. The role of orthodontic extrusive remodeling in the enhancement of soft and hard tissue profiles prior to implant placement: a systematic approach to the management of extraction site defects. Int J Periodontics Restorative Dent. 1993; 13(4):312-33.
15. Sclar AG The Bio-Col Technique. In: Sclar AG e. Soft tissue and esthetic considerations in implant dentistry. Quintessence Publishing Co., Inc. 2003; 75-112.

16. DA. G Immediate implant surgery: three-year retrospective evaluation of 50 consecutive cases. Int J Oral Maxillofac Implants. 1993; 8(4):388-99.

17. Gelb DA LR. Hierarchy of objectives in implant placement to maximize esthetics:use of pre-angulated abutments. Int J Periodontics Restorative Dent. 1993; 13(3):277-87.

18. Hammerle $\mathrm{CH}$ LN. Single stage surgery combining transmucosal implant placement with guided bone regeneration and bioresorbable materials. Clin Oral Implants Res. 2001; 12(1):9-18.

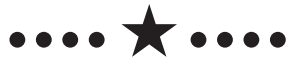

